\title{
Lueurs et leurres de la santé globale
}

À propos de MenAfriVac ${ }^{\circ}$ un vaccin « africain » contre la méningite

Oumy Thiongane, Janice Graham et Hélène Broutin

\section{(2) OpenEdition}

\section{Journals}

Édition électronique

URL : http://journals.openedition.org/anthropodev/607

DOI : 10.4000/anthropodev.607

ISSN : 2553-1719

Éditeur

APAD - Association pour l'anthropologie du changement social et du développement

\section{Édition imprimée}

Date de publication : 1 décembre 2017

Pagination : 213-234

ISBN : 979-10-93476-05-6

ISSN : 2276-2019

\section{Référence électronique}

Oumy Thiongane, Janice Graham et Hélène Broutin, «Lueurs et leurres de la santé globale »

Anthropologie \& développement [En ligne], 46-47 | 2017, mis en ligne le 01 juin 2018, consulté le 02 mai 2019. URL : http://journals.openedition.org/anthropodev/607 ; DOI : 10.4000/anthropodev.607

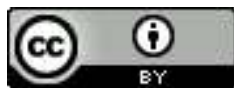

La revue Anthropologie \& développement est mise à disposition selon les termes de la Licence Creative Commons Attribution 4.0 International. 


\title{
Lueurs et leurres de la santé globale
}

\section{À propos de MenAfriVac ${ }^{\circledR}$, un vaccin « africain » contre la méningite}

\author{
Oumy Thiongane, Janice Graham, Hélène Broutin
}

Le projet Vaccins Méningite a permis à des millions d'Africains de disposer d'un vaccin peu coûteux, le MenAfriVac ${ }^{\circledR}$, grâce à un transfert de technologie. Les récits de la biomédecine en ont fait une histoire à succès, un mythe sociotechnique anonyme et collectif dans lequel le MenAfriVac ${ }^{\circledR}$ revêt une efficacité magique. Les promoteurs du vaccin ont choisi la rhétorique de l'élimination de la méningite par un vaccin, occultant un contexte épidémiologique plus complexe, notamment la persistance d'épidémies, documentées et dues à des sérogroupes autres que ceux ciblés par le MenAfriVac ${ }^{\circledR}$. Cette contribution a pour objectif d'analyser la construction $d u$ succès autour du vaccin et les paradoxes qui poussent à être prudent face aux initiatives de santé mondiale plébiscitant une solution universelle et expéditive par la technologie. Nous verrons que la solution par le vaccin repose sur des représentations clés de ce qu'est un succès. Ensuite, la contribution souligne que le MenAfriVac ${ }^{\circledR}$ est révélateur d'un type de "solution globale " privilégiant un émiettement des priorités et une visée à court terme de la santé publique.

In biomedical narratives, the Meningitis Vaccine Project (MVP) enabled the technology transfer for the development and implementation of MenAfriVac ${ }^{\circledR}$ vaccine at an affordable price for African countries. The presentation of MVP as a success story, however, may be more sociotechnical myth than fact. MenAfriVac ${ }^{\circledR}$ is far from being a "silver bullet". While successful in eliminating Meningitis A, MVP rhetoric highlighting meningitis control obscurred a more complex epidemiological context. This paper analyzes the discursive construction of success that supports global health single vaccine initiatives. 


\section{La vaccinologie à l'ère de la santé globale'}

"Because of a new meningitis vaccine, hundreds of millions of people no longer live in fear of the life-threatening infection, which wreaked havoc across Africa's so-called "meningitis belt" " (Dr Matshidiso Moeti and Dr Ala Alwan, 19 February 2016).

La première décennie du $21^{\text {ème }}$ siècle correspond à l'introduction accélérée de vaccins pédiatriques nouveaux ou améliorés dans les pays les plus pauvres grâce à des partenariats public-privé (PPP) impulsés notamment par la Fondation Bill et Melinda Gates (BMG) (Muraskin, 2005). D'autres mécanismes de financement tels que l'Alliance mondiale pour le vaccin et la vaccination (GAVI) et la Facilité internationale de financement pour la vaccination (IFFIm) ont été créés pour améliorer la disponibilité de ces vaccins dans des pays à faible ou moyen revenu (OMS, 2013 et 2006). Dès le début des années 2000 , la vaccinologie est ainsi entrée dans la santé globale que les anthropologues ont caractérisée à travers quatre régimes : sa dimension transnationale, son tournant biosécuritaire, son inscription dans le néolibéralisme, et l'échelle élargie des financements internationaux en direction des pays du Sud (Atlani-Duault et al., 2016 ; Lachenal, 2013 ; Lakoff, 2010).

Ce "grand moment» de la santé globale s'incarne par le développement, l'introduction et la mise à disposition de vaccins à travers des dispositifs sociotechniques transnationaux dans le cadre de projets portés par des consortiums. On peut citer parmi ces dispositifs : le Malaria Vaccine Development Program, le Malaria Vaccine Initiative, I'International AIDS Vaccine Initiative. Ils ont pour point commun d'ériger le vaccin comme innovation technologique et solution miracle permettant d'atteindre des objectifs ambitieux voire démesurés : le renforcement des

\footnotetext{
${ }^{1}$ Les données récoltées au Niger et au Sénégal ont bénéficié des financements du CRDI (Centre de recherche pour le développement international - Canada) via I'APHRC (African Population Health Research Centre), de l'Agence universitaire de la francophonie (AUF) et du PEERS (Programme d'excellence pour l'enseignement et la recherche au Sud - projet Thairenga). Nous remercions les coordonnateurs de ce numéro thématique ainsi que les relecteurs externes pour leurs commentaires et leurs suggestions lors de la lecture de ce texte.
} 
systèmes de santé, la réduction de la pauvreté ou des questions de biosécurité. Le Projet Vaccins Méningite (PVM) élaboré dans les années 2000 est une autre illustration de ce type de dispositif; il avait pour objectif de mettre à portée des pays africains frappés régulièrement par des épidémies meurtrières de méningite, un vaccin peu coûteux, à longue durée d'action.

\section{Les méningites et leurs vaccins : les limites de la prophylaxie}

Il existe trois types de méningite bactérienne à potentiel épidémique : celle causée par l'agent bactérien Haemophilus influenzae, globalement sous contrôle grâce à la vaccination incluse dans le Programme élargi de vaccination (PEV), celle due au méningocoque Neisseria meningitidis, et celle causée par le pneumocoque Streptococcus pneumoniae. Ces deux dernières continuent de représenter un problème majeur de santé publique en Afrique subsaharienne. La méningite à méningocoque provoque des épidémies meurtrières documentées depuis la fin du $18^{\text {ème }}$ siècle (Greenwood, 1999; Patterson et Hartwig, 1984). Elles apparaissent de façon saisonnière dans une bande sahélo-soudanienne appelée la « ceinture de la méningite » qui s'étend du Sénégal à l'Éthiopie (Lapeyssonnie, 1963). Les méningocoques sont des bactéries qui se trouvent chez l'être humain au niveau du rhinopharynx; environ $20 \%$ des individus sont porteurs asymptomatiques, c'est-à-dire qu'ils ne présentent pas de symptômes; ils ne sont pas non plus malades. Cette pathologie ${ }^{2}$ engage le pronostic vital en l'espace de vingt-quatre heures et reste mortelle en l'absence de traitement adéquat. La virulence du méningocoque et la non-prédicabilité des épidémies lui ont valu l'expression de " tueur sournois et imprévisible ». Malgré une saisonnalité de la méningite bien marquée et décrite, les démarrages épidémiques restent en effet localement imprévisibles (Lapeyssonnie, 1963).

\footnotetext{
${ }^{2}$ Signes principaux : soudaine fièvre, fortes céphalées pouvant s'accompagner de diarrhées, vomissements, éruption cutanée et raideur de la nuque - signe caractéristique très connu des populations africaines. Chez les nourrissons, le bombement de la fontanelle peut être un signe évocateur.
} 
Depuis les années 1970 , des vaccins polyosides ${ }^{3}$ appelés de manière narquoise des "pansements " ont été utilisés dans le cadre d'une stratégie "réactive " ou d'urgence pour circonscrire les épidémies (Saliou et Debois, 2002). Malgré cela, entre 1995 et 1996, l'épidémie a touché toute la " ceinture de la méningite " avec un bilan estimé à plus de 20000 morts (OMS, 2004 et 2002a). Plusieurs pays d'Afrique lui ont payé un lourd tribut. La stratégie "réactive ", consistant à vacciner une population minimale là où l'épidémie a été confirmée et déclarée, avait fait dès lors l'objet de critiques, le vaccin étant utilisé comme outil de contrôle au lieu de prévention (Higham, 1997 ; Kaninda et al., 1997 ; Perkins et al., 1997 ; Robbins et al., 1997). Les reproches faits à cette stratégie ont porté sur son manque de prise en compte des lourdeurs administratives et logistiques conduisant les pays pauvres à une posture d'impréparation permanente, son absence d'équité et le fait que les industriels pharmaceutiques refusent de mettre à disposition des pays des stocks de vaccins à titre préventif (Chippaux, 2001a et2001b; Robbins et al., 1997). Ce débat a aussi donné lieu à une querelle immunologique, le vaccin polyoside était critiqué pour son insuffisance à protéger les nourrissons et à offrir une immunité de longue durée (Perkins et al., 1997 ; Robbins et al., 2000). Cette controverse a conduit à reconsidérer la stratégie de santé publique autour des épidémies de méningite. Trois aspects ont prévalu après cette épidémie majeure : le renforcement de la surveillance bactériologique, la surveillance épidémiologique des nombres de cas et la mise à disposition d'une technologie vaccinale différente du polyoside (LaForce, 2003; Rosenstein et Perkins, 2003).

Le Projet Vaccins Méningite (PVM) a été mis en place à l'initiative de I'OMS (2002b). II avait pour objectif de mettre à disposition des pays les plus exposés aux épidémies, un vaccin conjugué contre la méningite due au sérogroupe $A$, à un prix abordable. Le vaccin conjugué est une association de deux matières premières : le polyoside $A$ qui est un sucre complexe et la toxine de tétanos ayant perdu ses propriétés microbiennes

\footnotetext{
${ }^{3}$ Les polyosides ou polysaccharides sont des glucides formés de sucres simples présents à la surface de certaines bactéries qui leur donnent leur potentiel pathogène. Les vaccins polyosides sont spécialement dirigés contre la capsule qui entoure ces bactéries.
} 
pour conserver des propriétés immunisantes. Cette association permet ainsi d'accroître la durée de la protection immunitaire.

Des travaux antérieurs sur les vaccins contre la méningite à méningocoque ont souligné l'importance d'interroger leur ontologie et la manière dont est construite leur compétence c'est-à-dire "la manière d'être au monde " du vaccin ainsi que les valeurs qui lui sont attribuées (Thiongane, 2013). Cette perspective inspirée des Sciences and Technologies Studies (STS) et que nous adoptons dans cet article, interroge les modalités par lesquelles le MenAfriVac ${ }^{\circledR}$, à travers le Projet Vaccins Méningite, s'est imposé comme solution aux épidémies de méningite (Latour, 2005).

\section{Cadre théorique et méthodologique}

Notre analyse s'appuie sur un large corpus de données obtenues à partir d'une collecte systématique d'informations ayant trait au développement du MenAfriVac ${ }^{\circledR}$. Le premier corpus de données provient du site du PVM ainsi que de celui de GAVI. Le second corpus concerne les données obtenues à partir d'ethnographies réalisées entre 2009 et 2015 dans trois pays aux contextes épidémiologiques différents : le Bénin, le Niger et le Sénégal. Lors de ces recherches, nous avons interviewé 99 personnes: personnel soignant, autorités de santé publique, hommes politiques, et populations. Au Niger, lors de l'épidémie de 2009, la recherche s'est déroulée au sein du comité national de gestion des épidémies, d'un centre de recherche sur les maladies infectieuses puis dans le district de Keita au nord-est du pays. Le Niger est frappé de manière dramatique et récurrente par les épidémies de méningite et les experts localisent le pays à l'épicentre de la "ceinture de la méningite " (Lapeyssonnie, 1963). Au Bénin, la recherche a concerné la région nord-est, dans l'Atacora, district de Cobly, zone d'épidémie modérée où fut introduit le MenAfriVac ${ }^{\circledR}$ en 2012. Au Sénégal, la recherche s'est faite dans la région centre, dans la zone rurale de Keur Socé où fut également introduit le MenAfriVac ${ }^{\circledR}$ en 2012. Le Sénégal a connu un recul drastique des épidémies depuis plusieurs années. 
Le troisième type de corpus concerne 1 ) une recension des articles scientifiques ayant trait à l'épidémiologie de la méningite et aux questions immunologiques, 2) ainsi qu'une analyse des bulletins de surveillance épidémiologique et bactériologique réalisés par les équipes de l'OMS de 2009 à 2014. Nous avons répertorié la littérature scientifique relative à l'épidémiologie de la méningite en Afrique de l'Ouest, et plus particulièrement les publications portant sur l'incidence des différents types de méningite. Nous avons ensuite analysé qualitativement, pour la période 2000 à 2010, le contenu des messages des auteurs par un processus itératif consistant à analyser les références données en bibliographie. Le codage des segments de données et des catégories a été réalisé à l'aide du logiciel NVIVO10. Les données sont utilisées de manière sélective dans cette contribution.

Notre approche est interdisciplinaire. Nous portons notre attention sur la construction des savoirs scientifiques et les controverses liées à la confrontation de paradigmes dans le domaine de l'épidémiologie, de la climatologie, de l'immunologie et de la santé publique via les grilles d'analyse de l'anthropologie des sciences et des techniques et de I'anthropologie du développement.

Le PVM entre dans le schéma d'une configuration développementaliste associant apport de ressources, de savoirs et de techniques par des hommes ou institutions en quête de transformation sociale (Olivier de Sardan, 1995). Il a été porté par des chercheurs, des industriels, des membres d'ONG, des politiques et des experts, ayant pour but de mettre à disposition des populations africaines de pays pauvres une ressource supplémentaire, un vaccin permettant de les protéger contre une maladie infectieuse hautement redoutable. Cette entreprise s'est faite en mettant à contribution des experts-bénéficiaires (chercheurs africains) dans leur lieu de vie (plateforme d'essais cliniques au Mali, en Gambie, au Sénégal) grâce à des ressources considérables (financement de la fondation BMG s'élevant à 70 millions de dollars pour l'étude préclinique).

Notre propos va s'articuler également autour des réflexions de l'anthropologue David Mosse qui constate que le succès d'un programme de développement ne se jauge pas seulement sur les indicateurs d'évaluation et les mesures de la performance, mais surtout sur la façon 
dont des interprétations particulières, des représentations, sont élaborées et soutenues socialement. II ne s'agit pas seulement de ce que le projet "fait " mais "comment et à qui il s'adresse ". Le succès dépend, premièrement, du fait d'établir une interprétation irrésistible des événements, deuxièmement, de maintenir cette représentation clé et, troisièmement, de conquérir ou d'impliquer un réseau de supporters ainsi que leur agenda (Mosse, 2005).

Nous allons d'abord présenter les protagonistes du projet vaccinal, avant d'examiner quelques postulats ou représentations clés construits et maintenus par le PVM, tout en les confrontant avec d'autres contre-récits dont le projet a fait peu de cas jusqu'ici. Nous analyserons par la même occasion comment ces représentations clés se sont construites et maintenues.

Entre philantrocapitalisme et dessein d'élimination : des acteurs et un vaccin emblématiques des projets de santé globale

L'analyse des comptes rendus de réunions organisées par l'OMS et ayant abouti à une série de décisions au début des années 2000 débouche sur une interrogation : comment penser les volte-face et la désarticulation entre demande sociale de produits pharmaceutiques et solutions prêtes à l'emploi qui ne cadrent guère avec les problèmes identifiés auparavant ?

Déjà ébranlés par la pandémie de méningite - qui se propage en Asie et en Europe aussi bien qu'en Afrique - au milieu des années 1990, les pays africains subissent une nouvelle hécatombe en 2002. Plus de 12000 cas suspects de méningite et 1500 morts sont par exemple répertoriés par les services sanitaires burkinabés. Les quelques prélèvements effectués soulignent qu'ils sont dus au sérotype W135 dont la circulation en Afrique était encore insuffisamment documentée. Ce sérotype, après avoir causé deux ans plus tôt une épidémie de méningite à La Mecque, avait été identifié dans quelques pays européens puis en Afrique de l'Ouest (Lingappa et al., 2003).

Sous le leadership de l'OMS, une consultation d'urgence sera organisée en septembre 2002 à Ouagadougou, capitale du Burkina Faso, durant laquelle il est conclu dans le communiqué suivant : 
"Les experts sont convenus que le plus important était d'obtenir un vaccin pouvant être utilisé contre toutes les futures flambées de méningite en Afrique. Ce vaccin devra couvrir les trois souches différentes de bactérie responsables des flambées antérieures y compris la nouvelle souche W135 et qui pourrait être à l'origine de nouvelles épidémies dès la fin de 2002 »(OMS, 2002a).

Or, le MenAfriVac ${ }^{\circledR}$ vaccin méningite contre le sérogroupe $A$ va être proposé aux États africains comme la meilleure solution aux épidémies de méningite saisonnières, malgré la diffusion de méningites à d'autres sérogroupes et l'existence sur le marché européen de vaccins les ciblant (Delrieu et al., 2011; Mueller et al., 2006; Ramsay et al., 2001). Néanmoins, restée longtemps une maladie infectieuse épidémique combattue par les institutions sanitaires sous le mode d'action d'urgence, cette nouvelle épidémie aboutit à une remobilisation des acteurs traditionnels œuvrant pour son contrôle (OMS, UNICEF, Centers for Disease Control and Prevention (CDC), Médecins sans frontières, Groupe de coordination internationale pour l'approvisionnement des vaccins antiméningococciques ou ICG, Agence de médecine préventive et institutions sanitaires africaines). Le Dr Marc LaForce, ancien officier des $C D C$, mobilisé en faveur du développement d'un vaccin conjugué dès la pandémie des années 1990, plaide à nouveau pour mettre à disposition un vaccin à bas coût en direction des pays africains (LaForce, 2003). Plusieurs tentatives de négociations menées par l'OMS avec une poignée de firmes pharmaceutiques du Nord spécialisées dans la fabrication des vaccins ont déjà échoué. L'objectif était de trouver un accord, soit pour faire baisser le prix des vaccins existants inaccessibles pour les malades africains, soit pour en produire un nouveau pour ce marché. La réunion de Ouagadougou est une occasion pour proposer une technologie vaccinale alternative discutée à plusieurs occasions en amont de cette consultation par les protagonistes du naissant PVM mis en place par la fondation BMG. Ce projet est le mobile d'un partenariat public-privé (PPP) regroupant I'OMS, le Programme for Appropriate Technology in Health (PATH) et le Serum Institute of India Limited (SIIL - une industrie pharmaceutique), avec la collaboration des CDC, de l'institut de santé publique norvégien et de SynCo Bio Partners, un laboratoire pharmaceutique situé aux Pays-Bas. Plusieurs plateformes d'essais cliniques Sud-Sud (Inde, Mali, Gambie, Sénégal) viendront renforcer le dispositif. 
PATH est une organisation internationale non lucrative, née pendant la deuxième moitié des années 1970 . Elle a son siège à Seattle, comme nombre d'ONG et d'organisations philanthropiques telles que la fondation BMG. À ses débuts, l'organisation est spécialisée dans les technologies reproductives telles que les contraceptifs, les condoms féminins et autres outils intra-utérins, puis commence à élargir le cercle des technologies délivrées aux populations des pays pauvres. Les décennies qui vont suivre vont amener au cœur de son action les technologies de santé à bas coût conçues comme de "puissants outils" destinés à sauver des vies (matériels de diagnostic, médicaments, puis vaccins). Principale tête de pont de la task force pour l'introduction du vaccin contre l'hépatite $B$ dans le PEV dès le milieu des années 1980, PATH acquiert alors une image de marque dans la contribution à la réduction de la mortalité maternelle et infantile.

D'autres acteurs institutionnels, comme SIIL, renforcent le dispositif du PPP. SIIL est une firme pharmaceutique familiale indienne appartenant au Poonawalla Group. Cette société est dirigée par Cyrus Poonawalla, un riche propriétaire terrien et entrepreneur indien qui décide avec son frère en 1966 de diversifier ses activités en créant SIIL. Elle s'illustrera par la production du vaccin antitétanique avant de devenir l'un des leaders mondiaux de fabrication de vaccins pédiatriques. En 1994, SIIL obtient son accréditation de I'OMS et approvisionne les agences onusiennes en vaccins. Le rôle de SIIL dans la fabrication du MenAfriVac ${ }^{\circledR}$ est de produire l'anatoxine tétanique. La particularité de la firme est de fabriquer des vaccins à bas coût. Le MenAfriVac ${ }^{\circledR}$ est négocié à 50 centimes de dollar la dose, élément clé de la success story du PVM. Par ailleurs, la firme est assurée par ce partenariat de régner sur le marché africain de la méningite pour une durée de dix ans ${ }^{4}$.

Le laboratoire SynCo Bio Partners, de son côté, est spécialisé dans la production de produits pharmaceutiques et technologiques non brevetés ou à contrat non contraignant. II est chargé de produire le polyoside $A$ qui, associé avec l'anatoxine tétanique, booste la mémoire immunitaire,

${ }^{4}$ Le prix du vaccin fixé peut varier en dehors de ce marché spécifique. 
constituant ainsi la conjugaison du vaccin. Après quelques déconvenues et retards, ce procédé sera obtenu en 2003 grâce à deux experts du vaccin : Robert Lee et Carl Frasch via le Centre de l'évaluation biologique et de la recherche appartenant à la Food and Drug Administration (FDA) américaine.

Dans l'imaginaire collectif, le PVM constitue un modèle de rupture en vaccinologie, associant secteur privé et secteur public et ayant surtout pu imposer un produit pharmaceutique à un prix abordable pour les pays pauvres en s'appuyant sur un transfert de technologie, défiant ainsi les modèles pharmaceutiques fondés sur l'exclusivisme et la fermeture. Nous allons analyser comment les multiples récits du PVM sont émaillés d'ambiguïtés qui ont souvent dissimulé l'accent mis sur une solution à court terme privilégiée par ces acteurs de santé globale. Nous allons pour cela nous baser sur deux représentations clés autour du projet: celle concernant la primauté d'un type de méningite et celle concevant et érigeant le MenAfriVac ${ }^{\circledR}$ en une solution magique lui conférant un caractère mythique.

Deux affirmations clés pour instaurer le récit d'un succès

\section{Le méningocoque $A$ est le premier agent bactérien responsable des épidémies de méningite en Afrique}

Les promoteurs du MenAfriVac ${ }^{\circledR}$ ont soutenu, durant le processus de développement du vaccin, la primauté du méningocoque $A$ comme cause principale des épidémies de méningite en Afrique (LaForce et Okwo-Bele, 2011). Analysons si cette assertion est soutenue par les publications scientifiques et comment elle a pu se maintenir dans le temps.

Dans les années 2000, l'OMS préconise le renforcement de la surveillance, la généralisation des tests bactériologiques et l'établissement d'un réseau de laboratoires nationaux. En 2002, elle décide de mettre en place une équipe d'épidémiologistes à la tête de laquelle elle place l'ancien directeur des statistiques, de la surveillance et des ripostes du Niger, qui est par la même occasion chargé de l'introduction du MenAfriVac ${ }^{\circledR}$ en Afrique. Cette équipe d'épidémiologistes appelés " équipe de support inter-pays " est regroupée au sein du Centre de surveillance pluri- 
pathologique de Ouagadougou. Ce centre est chargé de former les agents des laboratoires nationaux à la surveillance de la maladie, de collecter les données envoyées par ceux-ci et de les analyser. Elles sont ensuite comparées puis publiées dans un bulletin hebdomadaire diffusé largement. Elles concernent 18 pays de la " ceinture de la méningite ", en plus de la République Démocratique du Congo, placés sous surveillance renforcée et requérant par conséquent toute l'attention de cette équipe de l'OMS.

Des données empiriques au Niger ont montré que la majorité des malades vivent dans les campagnes ou dans des zones semi-urbaines comme Keita situé à $750 \mathrm{~km}$ de Niamey (Thiongane, 2013). Les conditions de réalisation d'une surveillance fiable par un diagnostic bactériologique sont précaires et peu de prélèvements parviennent aux laboratoires; les périodes d'épidémie suscitant le débordement des services de soins et l'affaiblissement du système de santé (Gruénais, 2001). De plus, les patients font majoritairement partie des cas suspects et seulement $10 \%$ des cas reportés sont testés (Agier et al., 2017). Ensuite, fait intrigant et peu interrogé par les experts de la méningite, près de $50 \%$ des prélèvements continuent d'être négatifs. Néanmoins, ils sont traités dans l'urgence comme des méningites au niveau des districts. En 2009, l'année où l'épidémie de méningite bat un record en Afrique de l'Ouest, l'équipe de support inter-pays déclare laconiquement :

"Les pays devraient faire des efforts dans la collecte et l'analyse des LCR [liquides céphalo-rachidiens]. Seulement $53 \%$ des échantillons sont positifs et cela mérite réflexion. »

Dans le même temps, d'autres recherches pointent du doigt la complexité des dynamiques épidémiologiques. Entre 2005 et 2009, des chercheurs de l'Agence de médecine préventive et du Centre Muraz au Burkina Faso signalent par exemple la part inquiétante des pneumocoques et des méningocoques à W135 parmi les causes de méningite (Du Châtelet et al., 2005). Pour 2005, leur étude révèle que, sur les 409 patients dont l'agent étiologique a été identifié, $72 \%$ des méningites à Neisseria étaient des W135. Les pneumocoques sont ensuite le deuxième agent causal identifié. L'année suivante, après une surveillance de deux ans au niveau de Bobo Dioulasso, $44 \%$ de cas confirmés sont dus aux pneumocoques. Ces chercheurs décident de renforcer leurs propos en publiant de 
nouvelles données en 2009 où ils associent les observations faites par leurs collègues au Togo (Traore et al., 2009). De la même façon, en 2005, au Ghana, les chercheurs de l'Institut tropical suisse sont intrigués par leur découverte : les méningites à pneumocoques plus sévères que celles dues aux méningocoques surviennent sensiblement de la même manière et présentent un profil biologique et épidémiologique semblable. Cependant la méningite à pneumocoque est cinq fois plus létale et atteint son pic un ou deux mois avant les épidémies dues au méningocoque ce qui, selon les chercheurs, laisserait supposer que les facteurs influençant les infections ne sont pas strictement similaires (Leimkugel et al., 2005).

Ainsi, l'équipe du Dr LaForce introduit-elle le MenAfriVac ${ }^{\circledR}$ au mépris des complexités et défaillances de la surveillance et en dépit des difficultés pour ces statistiques à refléter la dynamique épidémiologique. L'année où fut introduit le MenAfriVac ${ }^{\circledR}, 70 \%$ des prélèvements positifs du CERMES relèvent du W135, confirmant la variabilité des étiologies de la méningite (Thiongane, 2013). L'étude ethnographique réalisée dans la commune de Cobly au Nord-Bénin a par exemple mis aussi en évidence une vaccination d'urgence contre le W135 quelques mois seulement avant que le PVM y introduise son vaccin. Dans le même temps, à Keur Socé au centre du Sénégal, un médecin dubitatif s'interroge sur la pertinence du MenAfriVac ${ }^{\circledR}$ comme instrument préventif alors que les rares échantillons de LCR prélevés durant les dernières années étaient dus à un autre type de méningite, le W135 (Ndour et al., 2011).

L'argument de la primauté des méningocoques $A$ ne reflète donc pas la diversité des opinions ni la variabilité de l'épidémiologie de l'ensemble des pays vaccinés. Des chercheurs soulignent que, à long terme, les stratégies de prévention de routine nécessitent l'accès à des vaccins polyvalents, plutôt qu'à un vaccin monovalent conjugué ciblant le A. Certains chercheurs mettront même en garde contre les risques d'accaparement des subventions pour la recherche par le projet PVM et la difficulté d'obtenir des financements pour des vaccins ciblant un autre sérogroupe si le besoin s'en faisait sentir (Mueller et al., 2006). Ces voix dissonantes n'auront que peu de poids par rapport aux voix emblématiques des hommes politiques et des scientifiques africains qui, instrumentalisés par

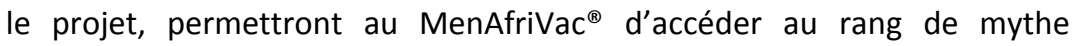
vaccinal. Récemment, dans son discours de départ, l'ancienne directrice de 
l'OMS, Margaret Chan, associait la fabrication du vaccin contre la méningite A à la "transformation sociale " de millions d'Africains et l'adjoint dans le bilan de santé publique de son organisation (OMS, 2017). Certaines déclarations faites après l'introduction du nouveau vaccin vont s'inscrire dans un discours "d'éradication de la méningite au Sahel ", entretenant la confusion (OMS, 2011 ; Sciences et Avenir, 2010). On peut supposer que le silence sur les autres sérotypes et, par conséquent, sur la complexité de l'épidémiologie de la méningite en Afrique, au profit d'une campagne d'information agressive, constituait l'un des moyens de distraire l'attention du public de l'approche simpliste du PVM.

\section{Le MenAfriVac ${ }^{\circledR}$ résulte d'une demande sociale}

$L^{\prime}$ érection du MenAfriVac ${ }^{\circledR}$ à un statut de produit pharmaceutique à haute valeur ajoutée s'est faite via un récit familier relayé à maintes reprises par ses promoteurs. Ce récit a été construit selon des séquences distinctes : 1 ) celle d'inquiétudes populaires cristallisées autour de l'accès à un vaccin abordable contre les méningites ; 2 ) celle de l'émergence et du transfert d'une "nouvelle» technologie permettant de répondre à cette inquiétude ; 3) et la prise en compte de nombreuses voix permettant au dispositif de s'inscrire dans une perspective de participation communautaire. L'une des trois modalités de la fabrique du succès des projets de développement concerne le réseau de supporters, c'est-à-dire les experts conseillers, les chercheurs et les officiels gouvernementaux qui participent également de la légitimation du projet (Mosse, 2005).

Le premier acte fondateur a été de faire du PVM la résultante d'une demande sociale pressante axée sur un prix modique. Les propos les plus emblématiques sont attribués au secrétaire général du ministre de la Santé nigérien de l'époque : Hassane Adamou. Ils ont été rapportés par le leader du PVM et viennent sous forme de supplique: "Please don't give us a vaccine we can't afford. That is worse than no vaccine ". Ces propos ont été repris par différentes plateformes scientifiques et médiatiques: la revue scientifique The Lancet, le magazine Science ou encore le site web du projet. Ils plébiscitent un vaccin dont le coût économique sera supportable pour des millions d'Africains même si des études ont montré que le coût modique d'une innovation vaccinale n'en garantit pas forcément son acceptabilité ; au contraire celle-ci peut être perçue comme un objet de 
santé inférieur (Guillermet et al., 2015) . Pour Marc LaForce, décrit comme "passionné ", ces propos justifient en quelque sorte ce qu'il considère comme une "mission", qui ne s'accomplit qu'à travers cette immunisation répétitive, forme d'acte de foi sur des millions de corps africains à la fois populations vulnérables et objets de conquête. "Mission " à l'issue de laquelle, Marc LaForce est décoré de la médaille d'or par l'Institut Sabin pour le vaccin comme dans un acte de canonisation, une anecdote révélatrice du processus et de la vision conquérante des projets de vaccination soutenus par les PPP et financés par les sociétés philanthropiques. Observateur de la reconfiguration de la vaccinologie et de la montée en puissance de la fondation Gates dans le domaine, William Muraskin notait combien, pour les fondateurs de l'organisation internationale PATH, acheminer des vaccins abordables aux plus pauvres relevait d'un impératif moral (Muraskin, 2005). L'instrumentalisation des propos d'Adamou à des fins de communication et la mission exaltée qu'ils déclenchent, rejoignent la grammaire des programmes de santé globale sur la vaccination en direction des pays du Sud conçus comme une "croisade " et constituent la prolongation de la vision messianique et obstinée de la médecine impériale européenne (Lachenal, 2014 ; Muraskin, 2005).

Après le " besoin » et la " mission ", la " participation ». À travers toute l'Afrique de l'Ouest, le même cérémonial inaugural renforçant la fiction de participation est mis à l'œuvre : au Burkina Faso, au Mali, au Niger, comme au Bénin, ce sont les présidents de la République parfois accompagnés de leurs épouses qui donnent le signal de l'ouverture des campagnes. Ces champions comme on les appelle dans le langage de la santé publique, choisis soigneusement pour faire changer les comportements en matière de prévention, administrent eux-mêmes la première injection à un jeune impressionné ou à un bébé cramponné au boubou de sa mère, tandis que des photographes et des caméramans de la presse populaire immortalisent le moment. Une poignée de courtiers de santé publique d'origine africaine, recrutés par le PVM et dont la biographie est exposée sur le site internet, sera en charge de mobiliser ce que Mosse appelle les "believers" (littéralement, les "croyants "). Les dirigeants politiques qui incarnent la tête de pont de ces croyants sont, depuis l'histoire de la variolisation, des figures de légitimation, des sponsors plébiscités par les projets 
d'immunisation de masse. Leur engouement pour des projets de santé publique est d'autant plus fort qu'il met en perspective leur capacité à gouverner la santé de leurs citoyens. L'histoire des sciences rappelle que le pacha d'Égypte comme Franklin Roosevelt aux États-Unis d'Amérique se sont servis respectivement de la vaccine et de la vaccination pour renforcer leur politique d'administration (Moulin, 2007). Le vaccin, instrument biopolitique, a servi de caution morale, maintenant l'illusion d'une participation populaire en réalité empiriquement infondée et structurée autour de la large consommation du MenAfriVac ${ }^{\circledR}$ comme produit marchandise par des millions de personnes.

La presse constituera immanquablement le relais de ces représentations du vaccin, et particulièrement du message ambigu du PVM contribuant à obscurcir et rendre invisible les autres méningites. Le 8 juin 2007, un communiqué de presse conjoint du consortium déclare : " Un meilleur vaccin contre la méningite pourrait marquer la fin de ce fléau mortel en Afrique ". L'OMS elle-même renchérit: "Un nouveau vaccin révolutionnaire contre la méningite promet de mettre fin aux épidémies en Afrique "; tandis que la banderole sur le site de PATH affiche pompeusement: "Revolutionnary vaccine to end Africa's meningitis epidemics ". Les services de communication de l'OMS-AFRO ont consacré ainsi une histoire attractive de la vaccination par le MenAfriVac ${ }^{\circledR}$, conçue même au-delà d'une "innovation ", comme une "révolution en matière de santé publique " (OMS, 2011b). L'analyse de David Mosse sur les activités d'un projet de développement, nous montre combien ces promesses convergentes sous forme de titres accrocheurs contribuent à renforcer la production du succès (Mosse, 2005). Elles donnent ainsi l'impression d'un changement radical, qui ne repose pas sur une base empirique. Le retour des épidémies de méningite dues au sérogroupe $C$, faisant plus de 600 morts au Niger en 2015, 1000 morts au Nigeria en $2017^{5}$, réinscrivent avec force le fossé entre les promesses dispro-

${ }^{5} \mathrm{Au}$ moment où nous rédigeons cet article, cette saison épidémique toujours en cours au Nigeria a touché plus de 14000 personnes. Des vaccins polyvalents sont en attente pour vacciner des millions de personnes à risque dans les 22 États touchés. Au Niger, l'épidémie continue de se développer avec 179 personnes tuées et 3037 cas suspects. 
portionnées du PVM et la vérité intangible du terrain et du corps humain qui relèvent respectivement de complexités épidémiologiques et immunologiques.

\section{Conclusion}

Le mythe, au sens anthropologique, se structure autour de récits fondateurs qui sont à la fois collectifs et anonymes. L'histoire des sciences a souligné à maintes reprises comment la médecine au $19^{\text {ème }}$ siècle a connu ses lettres de noblesse grâce à la découverte de vaccins dont chaque récit figé et épuré a permis de renforcer le mythe de Pasteur, figure légendaire intimement liée à leur triomphe (Latour, 2001 ; Moulin, 1996 et 1991). Aujourd'hui, les vaccins restent plus que jamais l'une des premières technologies par le biais desquelles les épidémies dues aux maladies infectieuses sont jugulées ou évitées. La santé publique y tire une grande partie de sa légitimité et de sa gloire, en dépit des peurs sur les effets iatrogènes des vaccins dans les pays du Nord (Larson et al., 2014). La légitimité de la vaccination repose majoritairement sur la métaphore médicale de l'immunité. Un terme ancien désignant l'exemption d'impôt ou de service militaire qui, en immunologie, définit la capacité d'un individu à être indemne d'une infection grâce à un vaccin, réinscrivant celui-ci au cœur des mythes scientifiques et nationaux (Baylac Paouly, 2015 ; Lambrichs, 1999). Les vaccins contre la méningite ne font pas exception $^{6}$ et le MenAfriVac ${ }^{\circledR}$ a ainsi été consacré comme solution miracle au service $d^{\prime}$ 'une transformation sociale nécessaire et souhaitable.

Les sciences sociales ont montré que les vaccins, instruments de biopolitique, sont des êtres techniques non neutres adossés à des enjeux politiques, économiques, travaillés par des intérêts mercantilistes (Graham, 2016; Wailoo et al., 2010). Le PVM, initiative sanitaire pro-

6 La vaccination contre la méningite de « 80 millions de Brésiliens», ou "l'épopée brésilienne ", dans les années 1970 a été la plus grande campagne de vaccination effectuée dans le monde, elle a donné lieu à des récits épiques sur la grandeur de l'industrie pharmaceutique française. Charles Mérieux et le médecin Léon Lapeyssonnie ont consacré plusieurs témoignages à cet épisode. 
mouvant une collaboration horizontale Sud-Sud, a révélé au bout du compte de quelle manière le montage des partenariats public-privé peut s'avérer fallacieux (Biehl, 2006). Un modèle de santé globale structuré de manière séduisante a primé ici sur les réels besoins des populations: rendre accessible un vaccin couvrant les différentes souches présentes et en finir avec la gestion de l'épidémie par l'urgence en réinscrivant la prévention au cœur de l'action de santé publique. Le MenAfriVac ${ }^{\circledR}$ est la résultante d'une histoire unique véhiculant des stéréotypes comme des antiennes à la messe, et dont cet article seul ne suffit pas à rendre compte. Or, les stéréotypes restent incomplets et parviennent difficilement à rendre intelligible la complexité des mondes sociaux (Mkhwanazi, 2016). À l'inverse du discours véhiculé sur le "potentiel d'éradication" de la méningite en Afrique, I'histoire des épidémies de méningite est une histoire émaillée par les incertitudes de la science, que la technologie est insuffisante à appréhender. L'entrée par l'analyse des représentations et de la production du succès d'un projet sociotechnique tel que le PVM, a pour intérêt de mettre en perspective les mécanismes dont un projet de développement et de santé publique se sert afin de stabiliser et sécuriser ses propres schémas d'interprétation.

\section{Bibliographie}

AGIER L., MARTINY N., THIONGANE O., MUELLER J.E., PAIREAU J., WATKINS E.R., IRVING T.J., KOUTANGNI T., BROUTIN H., 2017, " Towards understanding the epidemiology of Neisseria meningitidis in the African meningitis belt: a multi-disciplinary overview ", International Journal of Infectious Diseases, 54 : 103-112.

ATLANI-DUAULT L., DOZON J.P., WILSON A., DELFRAISSY J.F., MOATTI J.P., 2016, "State humanitarian verticalism versus universal health coverage: a century of French international health assistance revisited ", The Lancet, $387: 2250-2262$.

BAYLAC PAOULY B., 2015, " Histoire du vaccin antiméningococcique (1963-1973) : contextes de développement, système de contrainte et réseaux scientifiques ", mémoire de maîtrise sous la direction de Jonathan Simon, université Claude Bernard Lyon 1, Lyon : $59 \mathrm{p}$. 
BIEHL J., 2006, "Pharmaceutical governance ", in PETRYNA A., LAKOFF A., KLEINMAN A. (eds.), Global Pharmaceuticals: Ethics, Markets, Practices, Duke University Press : 206-239.

DU CHÂTELET I.P., TRAORE Y., GESSNER B.D., ANTIGNAC A., NACCRO B., NJANPOPLAFOURCADE B.M., OUEDRAOGO M.S., TIENDREBEOGO S.R., VARON E., TAHA M.K., 2005, "Bacterial Meningitis in Burkina Faso: Surveillance Using Field-Based Polymerase Chain Reaction Testing ", Clinical Infectious Diseases, 40 : 17-25.

CHIPPAUX, J.P., 2001a, "Épidémies au sud, vaccin au nord ", Sciences Au Sud, 1.

CHIPPAUX J.P., 2001b, "Conflits autour de la méningite en Afrique ", Le Monde Diplomatique, mai : 22.

DELRIEU I., YARO S., TAMEKLOE T.A.S, NJANPOP-LAFOURCADE B.M., TALL H., JAILLARD P., OUEDRAOGO M.S., BADZIKLOU K., SANOU O., DRABO A., GESSNER B.D., KAMBOU J.L., MUELLER J.E., 2011, "Emergence of epidemic Neisseria meningitidis serogroup $X$ meningitis in Togo and Burkina Faso », PloS One, 6(5).

GRAHAM J.E., 2016, «Ambiguous capture: Collaborative Capitalism and the Meningitis Vaccine Project », Medical Anthropology, 35(5) : 419-432.

GREENWOOD B., 1999, "Meningococcal meningitis in Africa ", Transaction of the Royal Society of Tropical Medicine and Hygiene, 93 : 341-353.

GRUÉNAIS M.É., 2001, "En Afrique, le sida réformateur? ", Nature Science et Sociétés, 9 : 9-17.

GUILLERMET E., DICKO H.M., MAI L.T.P., N'DIAYE M., HANE F., BA S.O., GOMIS K., THO N.T.T., LIEN N.T.P., THAN P.D., DINH T.V., JAILLARD P., GESSNER B.D., COLOMBINI A., 2015, "Acceptability and Feasibility of Delivering Pentavalent Vaccines in a Compact, Prefilled, Autodisable Device in Vietnam and Senegal », PLoS One, 10(7).

HIGHAM J.H., 1997, "Meningococcal vaccine in sub-Saharan Africa ", The Lancet, $350: 1707-1708$.

KANINDA A.V., VARAINE F., HENKENS M., PACKET C., 1997, « Meningococcal vaccine in sub-Saharan Africa ", The Lancet, 350.

LACHENAL G., 2013, "Le stade Dubaï de la santé publique », Revue Tiers Monde, $215: 53-71$. 
LACHENAL G., 2014, Le médicament qui devait sauver l'Afrique: Un scandale pharmaceutique aux colonies, La Découverte, Paris.

LAFORCE F.M., 2003, " Control of epidemic meningitis in sub-Saharan Africa: our solution is more practical and affordable ", Bulletin of World Health Organization, 81 : 753-755.

LAFORCE F.M., OKWO-BELE J.M., 2011, «Eliminating epidemic group A meningococcal meningitis in Africa through a new vaccine ", Health Affairs, 30 : 1049-1057.

LAKOFF A., 2010, "Two regimes of global health ", Humanity: An International Journal of Human Rights, Humanitarism, and Development, 1(1) : 59-79.

LAMBRICHS L.L., 1999, Virus passion, Paris, Robert Laffont.

LAPEYSSONNIE L., 1963, "La méningite cérébro-spinale en Afrique ", Bulletin de l'Organisation mondiale de la santé, 28(suppl. 1), 114 p.

LARSON H.J., JARRETT C., ECKERSBERGER E., SMITH D.M.D., PATERSON P., 2014, " Understanding vaccine hesitancy around vaccines and vaccination from a global perspective: a systematic review of published literature 2007$2012 »$, Vaccine, 32 : 2150-2159.

LATOUR B., 2001, Pasteur : guerre et paix des microbes, suivi de Irréductions, Paris, La Découverte.

LATOUR B., 2005, La science en action. Introduction à la sociologie des sciences, Paris, La Découverte.

LEIMKUGEL J., ADAMS FORGOR A., GAGNEUX S., PFLÜGER V., FLIERL C., AWINE E., NAEGELI M., DANGY J.P., SMITH T., HODGSON A., PLUSCHKE G., 2005, "An outbreak of serotype 1 Streptococcus pneumoniae meningitis in northern Ghana with features that are characteristic of Neisseria meningitidis meningitis epidemics ", Journal of Infectious Diseases, 192 : 192-199.

LINGAPPA J.R., AL-RABEAH A.M., HAJJEH R., MUSTAFA T., FATANI A., AL-BASSAM T., BADUKHAN A., TURKISTANI A., MAKKI S., AL-HAMDAN N. et al., 2003, "Serogroup W-135 meningococcal disease during the Hajj, 2000 ", Emerging Infectious Diseases, 9 : 665-671.

MKHWANAZI N., 2016, « Medical Anthropology in Africa: The Trouble with a Single Story ", Medical Anthropology, 35 : 193-202. 
MOSSE D., 2005, Cultivating Development: An Ethnography of Aid Policy and Practice, London, Pluto Press Anthropology, Culture and Society Series.

MOULIN A.M., 1996, L'Aventure de la vaccination, Paris, Fayard.

MOULIN A.M., 1991, Le dernier langage de la médecine : histoire de l'immunologie de Pasteur au sida, Paris, Presses universitaires de France.

MOULIN A.M., 2007, "Les vaccins, l'État moderne et les sociétés ", Médecine Science, $23:$ 428-434.

MUELLER J.E., BORROW R., GESSNER B.D., 2006, « Meningococcal serogroup W135 in the African meningitis belt: epidemiology, immunity and vaccines", Expert Review Vaccines, 5 : 319-336.

MURASKIN W., 2005, Crusade to Immunize the World's Children, Lulu.com.

NDOUR C.T., MANGA N.M., DIA M., CAMARA B., CISSÉ F., 2011, "Neisseria meningitidis serogroup W135 meningitis in Senegal from 2000 to 2009 ", Médecine et Maladies Infectieuses, 41 : 495-497.

OLIVIER DE SARDAN J.P., 1995, Anthropologie et développement. Essai en socioanthropologie du changement social, Paris, Karthala.

OMS, 2002a, "Prevention and control of epidemic meningitis control disease in Africa ", Report of WHO technical consultation meeting, Genève, WHO.

OMS, 2002b, "Appel en faveur de mesures d'urgence contre la méningite en Afrique ", Ouagadougou, 27 septembre 2002, http://www.who.int /mediacentre/news/releases/pr71/fr, consulté le 27 juillet 2015.

OMS, 2004, "Meeting the public health challenge of epidemic meningitis in Africa », http://www.who.int/csr/disease/meningococcal/challenge2004 _11_10/en

OMS, 2006, "Malaria vaccine technology roadmap ", http://www.who.int /immunization/sage/meetings/2013/april/7_Malaria_Vaccine_TRM_Final .pdf?ua $=1$

OMS, 2011a, "Méningite : la fin d'un fléau centenaire ? », Bulletin OMS, 89 : 550551.

OMS, 2011b, « Introduction du nouveau vaccin conjugué contre la méningite "A". Une révolution en matière de santé publique", http://who.insomnation.com/sites/default/files/pdf/Burkina-FasoMenAfriVac-juin2011-fr.pdf 
OMS, 2013, "Malaria vaccine technology roadmap Update », http://www.who.int /immunization/sage/meetings/2013/april/6_Draft_roadmap_update_v_5 _March.pdf?ua=1

OMS, 2017, "Allocution du Dr Margaret Chan à la Soixante-Dixième Assemblée mondiale de la Santé ", http://www.who.int/dg/speeches/2017/addressseventieth-assembly/fr

PATH, 2012, "Honoring a pioneer in vaccine development », http:// www.path.org/news/press-room/182

PATTERSON K.D., HARTWIG G.W., 1984, Cerebrospinal meningitis in West Africa and Sudan in the twentieth century, Los Angeles, Crossroads Press.

PERKINS B.A., BROOME C.V., ROSENSTEIN N.E., SCHUCHAT A., REINGOLD A.L., 1997, "Meningococcal vaccine in sub-Saharan Africa ", The Lancet, 350 : 1708 ; author reply : 1709-1710.

RAMSAY M.E., ANDREWS N., KACZMARSKI E.B., MILLER E., 2001, « Efficacy of meningococcal serogroup $C$ conjugate vaccine in teenagers and toddlers in England », The Lancet, 357 : 195-196.

ROBBINS J.B., SCHNEERSON R., GOTSCHICH E.C., 2000, " A rebuttal: epidemic and endemic meningococcal meningitis in sub-Saharan Africa can be prevented now by routine immunization with group $A$ meningococcal capsular polysaccharide vaccine ", Pediatric Infectious Diseases Journal, $19: 945$.

ROBBINS J.B., TOWNE D.W., GOTSCHLICH E.C., SCHNEERSON R., 1997, " "Love's labours lost": failure to implement mass vaccination against group $A$ meningococcal meningitis in sub-Saharan Africa ", The Lancet, $350: 880$ 882.

ROSENSTEIN N.E., PERKINS B.A., 2003, "Conjugate meningococcal vaccines offer a much more promising alternative ", Bulletin of World Health Organization, $81: 752$.

SALIOU P., DEBOIS H., 2002, "Quelles stratégies vaccinales contre les épidémies africaines de méningite à méningocoque ? ", Bulletin de la Société de Pathologie Exotique, 95 : 326-330.

SCIENCES ET AVENIR, 2010, « Un nouveau vaccin pour éradiquer la méningite au Sahel », https://www.sciencesetavenir.fr/sante/un-nouveau-vaccin-poureradiquer-la-meningite-en-afrique_25465 
THIONGANE O.B., 2013, "Anthropologie de la méningite au Niger. Espaces épidémiques, mobilisations scientifiques et conceptions de la maladie ", thèse de doctorat sous la direction de Mahaman Tidjani Alou et Anne Marie Moulin, Paris, École des Hautes Études en Sciences Sociales, 414 p.

TRAORE Y., TAMEKLO T.A., NJANPOP-LAFOURCADE B.M., LOURD M., YARO S., NIAMBA D., DRABO A., MUELLER J.E., KOECK J.L., GESSNER B.D., 2009, "Incidence, seasonality, age distribution, and mortality of pneumococcal meningitis in Burkina Faso and Togo ", Clinical Infectious Diseases, 48(suppl. 2) : S181-189.

WAILOO K., LIVINGSTON J., EPSTEIN S., ARONOWITZ R., 2010, Three Shots at Prevention: The HPV Vaccine and the Politics of Medicine's Simple Solutions, Johns Hopkins University Press, Baltimore.

\section{Aiviviv}

Oumy Thiongane est chercheure associée en anthropologie

Dalhousie University, Technoscience \& Regulation Research Unit (Canada)

et IRD, Antenne Bobo Dioulasso (Burkina Faso)

E-mail : o.moirouxthiongane@gmail.com

Janice Graham est professeure en anthropologie et pédiatrie

Dalhousie University, Technoscience \& Regulation Research Unit (Canada)

E-mail : janice.graham@dal.ca

Hélène Broutin est chargée de recherche CNRS en écologie des maladies infectieuses

MIVEGEC, UMR CNRS-IRD-université de Montpellier (France)

et université Cheikh Anta Diop (UCAD), faculté de médecine, Dakar (Sénégal)

E-mail : helene.broutin@ird.fr 